\title{
A Topology on Milnor's Group of a Topological Field and Continuous Joint Determinants
}

\author{
Sung Myung \\ Department of Mathematics Education, Inha University, 253 Yonghyun-dong, Nam-gu, Incheon 402-751, Republic of Korea
}

Correspondence should be addressed to Sung Myung; s-myung1@inha.ac.kr

Received 28 September 2017; Accepted 26 November 2017; Published 12 December 2017

Academic Editor: Daniel Simson

Copyright (c) 2017 Sung Myung. This is an open access article distributed under the Creative Commons Attribution License, which permits unrestricted use, distribution, and reproduction in any medium, provided the original work is properly cited.

\begin{abstract}
For the tuple set of commuting invertible matrices with coefficients in a given field, the joint determinants are defined as generalizations of the determinant map for the square matrices. We introduce a natural topology on Milnor's $K$-groups of a topological field as the quotient topology induced by the joint determinant map and investigate the existence of a nontrivial continuous joint determinant by utilizing this topology, generalizing the author's previous results on the continuous joint determinants for the commuting invertible matrices over $\mathbb{R}$ and $\mathbb{C}$.
\end{abstract}

\section{Introduction}

In [1], a joint determinant is introduced as a generalization of the determinant map for invertible matrices. More precisely, for a field $k$, a joint determinant $D\left(=D_{l}\right)(l \geq 1)$ is defined as a map from the set of $l$-tuples of commuting matrices in $\mathrm{GL}_{n}(k)(n \geq 1)$ into some abelian group $(G,+)$ which satisfies the following properties.

(i) Multilinearity: for $l+1$ commuting matrices $A_{1}$, $\ldots, A_{l}$ and $B$ in $\mathrm{GL}_{n}(k)$ for some $n \geq 1$, we have $D\left(A_{1}, \ldots\right.$, $\left.A_{i} B, \ldots, A_{l}\right)=D\left(A_{1}, \ldots, A_{i}, \ldots, A_{l}\right)+D\left(A_{1}, \ldots, B, \ldots, A_{l}\right)$.

(ii) Block diagonal matrices: for commuting $A_{1}, \ldots, A_{l} \in$ $\mathrm{GL}_{m}(k)$ and commuting $B_{1}, \ldots, B_{l} \in \mathrm{GL}_{n}(k)$ for some $m, n \geq$ 1 , we have

$$
\begin{aligned}
D & \left(\left(\begin{array}{cc}
A_{1} & 0 \\
0 & B_{1}
\end{array}\right), \ldots,\left(\begin{array}{cc}
A_{l} & 0 \\
0 & B_{l}
\end{array}\right)\right) \\
& =D\left(A_{1}, \ldots, A_{l}\right)+D\left(B_{1}, \ldots, B_{l}\right) .
\end{aligned}
$$

(iii) Similar matrices: for commuting matrices $A_{1}, \ldots$, $A_{l} \in \mathrm{GL}_{n}(k)$ and any $S \in \mathrm{GL}_{n}(k)$, we have $D\left(S A_{1} S^{-1}, \ldots\right.$, $\left.S A_{l} S^{-1}\right)=D\left(A_{1}, \ldots, A_{l}\right)$.

(iv) Polynomial homotopy: for commuting $A_{1}(t), \ldots$, $A_{l}(t) \in \mathrm{GL}_{n}(k[t])$, we have $D\left(A_{1}(0), \ldots, A_{l}(0)\right)=D\left(A_{1}(1)\right.$, $\left.\ldots, A_{l}(1)\right)$.

$$
\begin{aligned}
& \text { Using the standard inclusion } \mathrm{GL}_{n}(k) \hookrightarrow \mathrm{GL}_{n+1}(k) \\
& \qquad\left(\begin{array}{ccc}
a_{11} & \cdots & a_{1 n} \\
\vdots & \ddots & \vdots \\
a_{n 1} & \cdots & a_{n n}
\end{array}\right) \longmapsto\left(\begin{array}{cccc}
a_{11} & \cdots & a_{1 n} & 0 \\
\vdots & \ddots & \vdots & 0 \\
a_{n 1} & \cdots & a_{n n} & 0 \\
0 & \cdots & 0 & 1
\end{array}\right)
\end{aligned}
$$

we define $\mathrm{GL}(k)$ as the direct limit of these groups $\mathrm{GL}(k)=$ $\cup_{n \rightarrow \infty} \mathrm{GL}_{n}(k)$. Using the above inclusions, we may identify the direct limit $\operatorname{Comm}_{l}(k)$ of the set of $l$-tuples of commuting matrices in $\mathrm{GL}_{n}(k)^{l}=\mathrm{GL}_{n}(k) \times \cdots \times \mathrm{GL}_{n}(k)$ over $n$ as a subset of $\mathrm{GL}(k)^{l}=\mathrm{GL}(k) \times \cdots \times \mathrm{GL}(k)$. Then, a joint determinant may be thought of as a map from $\operatorname{Comm}_{l}(k)$ into an abelian group $G$.

The main result in [1] about the joint determinants is that there exists a one-to-one correspondence between the set of joint determinants from $\operatorname{Comm}_{l}(k)$ into an abelian group $G$ and the set of group homomorphisms from Milnor's $K$-group $K_{l}^{M}(k)$ into $G$. Milnor's $K$-group is introduced in [2] as the quotient group of the tensor product $k^{*} \otimes \cdots \otimes k^{*}$ by the subgroup generated by elements of the form $a_{1} \otimes \cdots \otimes a_{l}$, where $a_{i}+a_{j}=1$ for some $i, j(1 \leq i<j \leq l)$. It is a major object of study in algebraic $K$-theory and appears in numerous literatures. For example, Voevodsky's proof of 
Bloch-Kato conjecture [3] relates Milnor's $K$-group of a field with its étale cohomology. The element of $K_{l}^{M}(k)$ represented by $a_{1} \otimes \cdots \otimes a_{l}$ is typically denoted by a symbol $\left\{a_{1}, \ldots\right.$, $\left.a_{l}\right\}$.

To describe the "universal" joint determinant $\operatorname{Comm}_{l}(k) \rightarrow K_{l}^{M}(k)$, we need the Goodwillie group $\mathrm{GW}_{l}(k)$ which is defined to be the abelian group generated by $l$-tuples of commuting matrices $\left(A_{1}, \ldots, A_{l}\right)\left(A_{1}, \ldots, A_{l} \in \mathrm{GL}_{n}(k)\right.$ for various $n \geq 1$ ), subject to the following 4 kinds of relations.

(i) Identity matrices: $\left(A_{1}, \ldots, A_{l}\right)=0$ when $A_{i}$ for some $i$ is equal to the identity matrix $I_{n} \in \mathrm{GL}_{n}(k)$.

(ii) Similar matrices: $\left(A_{1}, \ldots, A_{l}\right)=\left(S A_{1} S^{-1}, \ldots, S A_{l} S^{-1}\right)$ for commuting $A_{1}, \ldots, A_{l} \in \mathrm{GL}_{n}(k)$ and any $S \in \mathrm{GL}_{n}(k)$.

(iii) Direct sum: $\left(A_{1}, \ldots, A_{l}\right)+\left(B_{1}, \ldots, B_{l}\right)=\left(\left(\begin{array}{cc}A_{1} & 0 \\ 0 & B_{1}\end{array}\right)\right.$, $\left.\ldots,\left(\begin{array}{cc}A_{l} & 0 \\ 0 & B_{l}\end{array}\right)\right)$ for commuting $A_{1}, \ldots, A_{l} \in \mathrm{GL}_{n}(k)$ and commuting $B_{1}, \ldots, B_{l} \in \mathrm{GL}_{m}(k)$.

(iv) Polynomial homotopy: $\left(A_{1}(0), \ldots, A_{l}(0)\right)=\left(A_{1}(1)\right.$, $\left.\ldots, A_{l}(1)\right)$ for commuting matrices $A_{1}(t), \ldots, A_{l}(t)$ in $\mathrm{GL}_{n}(k[t])$, where $k[t]$ is the polynomial ring over $k$ with the indeterminate $t$.

The universal joint determinant map $\Phi_{l}: \operatorname{Comm}_{l}(k) \rightarrow$ $K_{l}^{M}(k)$ is then the composite of the natural map $\operatorname{Comm}_{l}(k) \rightarrow$ $\mathrm{GW}_{l}(k)$, which sends an $l$-tuple of commuting matrices to a generator of $\mathrm{GW}_{l}(k)$ and the isomorphism $\phi_{l}: \mathrm{GW}_{l}(k) \stackrel{\sim}{\rightarrow}$ $K_{l}^{M}(k)$, which is described in the proof of Theorem 6.7 of [1]. From the fact that $\phi$ is an isomorphism follows easily the oneto-one correspondence between the set of joint determinants from $\operatorname{Comm}_{l}(k)$ into an abelian group $G$ and the set of group homomorphisms from Milnor's $K$-group $K_{l}^{M}(k)$ into G.

When $l=1, \mathrm{GW}_{l}(k) \simeq k^{*}$ and the universal joint determinant is nothing but the traditional determinant map (Proposition 4.4 of [1]).

The definition of joint determinant maps is given in purely algebraic terms and so there are possibilities of very complicated joint determinants; for example, when $k$ is the field $\mathbb{C}$ of complex numbers or $\mathbb{R}$ of real numbers, Milnor's $K$-groups $K_{l}^{M}(k)$ for $l \geq 2$ are known to be uniquely divisible or a direct sum of a cyclic group of order 2 and a uniquely divisible group, respectively [2].

Thus, if we disregard the topological continuity of a joint determinant map, the joint determinants are far from trivial, but if we require a joint determinant to be continuous, then the situation becomes drastically different. It is proven that, for $l \geq 2$, there exists only one nontrivial joint determinant from $\operatorname{Comm}_{l}(\mathbb{R})$ into $\mathbb{R}^{\times}$, which is continuous when restricted to the set of commuting matrices in $\mathrm{GL}_{n}(\mathbb{R})$, for each $n$, with the standard topology (Corollary 7.3 of [1]).

In the present article, we generalize this result to determine all possible continuous joint determinants from $\operatorname{Comm}_{l}(\mathbb{R})$ or $\operatorname{Comm}_{l}(\mathbb{C})$ to a topological abelian group $G$. For this purpose, we introduce a natural topology on Milnor's $K$-groups $K_{l}^{M}(k)$ for a topological field $k$ as the quotient topology induced by the joint determinant map and show that, in case of $k=\mathbb{R}$ or $\mathbb{C}$, the natural topology on $K_{l}^{M}(k)$ is disjoint union of two indiscrete components or indiscrete topology, respectively. This indicates that, for $k=\mathbb{R}$ or $\mathbb{C}$, the "universal" continuous joint determinant turns out to be $\operatorname{Comm}_{l}(\mathbb{R}) \rightarrow \mathbb{Z}_{2}$ or $\operatorname{Comm}_{l}(\mathbb{C}) \rightarrow\{1\}$, respectively.

\section{A Natural Topology on $K_{l}^{M}(k)$}

For a topological field $k, \mathrm{GL}(k)=\cup_{n \rightarrow \infty} \mathrm{GL}_{n}(k)$ is a topological group with the direct limit topology, that is, a subset $U$ of $\mathrm{GL}(k)$ is open if and only if $U \cap \mathrm{GL}_{n}(k)$ is open for each $n \geq 1$ (e.g., 3.1 of [4]). The topology on $\operatorname{Comm}_{l}(k)$ is given by the subspace topology regarding it as a subspace of the product space $\mathrm{GL}(k)^{l}=\mathrm{GL}(k) \times \cdots \times \mathrm{GL}(k)$. Then it coincides with the direct limit topology if we think of $\mathrm{Comm}_{l}(k)$ as the direct limit of the subspace of $l$-tuples of commuting matrices in the space $\mathrm{GL}_{n}(k)^{l}=\mathrm{GL}_{n}(k) \times \cdots \times$ $\mathrm{GL}_{n}(k)$ over $n$.

Definition 1. For a topological field $k$, the topology on Milnor's $K$-group $K_{l}^{M}(k)$ is the quotient topology with respect to the map $\Phi_{l}: \operatorname{Comm}_{l}(k) \rightarrow K_{l}^{M}(k)$, which is the composite of a natural map $\operatorname{Comm}_{l}(k) \rightarrow \mathrm{GW}_{l}(k)$ followed by the group isomorphism $\phi_{l}: \mathrm{GW}_{l}(k) \stackrel{\sim}{\rightarrow} K_{l}^{M}(k)$ which is described in the proof of Theorem 6.7 of [1].

The obvious map $\operatorname{Comm}_{l}(k) \rightarrow \mathrm{GW}_{l}(k)$ is actually surjective by Corollary 4.3 of [1] and so $\Phi_{l}$ is a surjection.

Theorem 2. $K_{l}^{M}(k)$ is a topological group with respect to the topology given in Definition 1.

Proof. By the definition of the Goodwillie group $\mathrm{GW}_{l}(k)$, the group law on $K_{l}^{M}(k)$ is given via $\phi_{l}: \mathrm{GW}_{l}(k) \stackrel{\sim}{\rightarrow}$ $K_{l}^{M}(k)$ by the direct sum rule: $\left(A_{1}, \ldots, A_{l}\right)+\left(B_{1}, \ldots, B_{l}\right)=$ $\left(\left(\begin{array}{cc}A_{1} & 0 \\ 0 & B_{1}\end{array}\right), \ldots,\left(\begin{array}{cc}A_{l} & 0 \\ 0 & B_{l}\end{array}\right)\right)$ for commuting $A_{1}, \ldots, A_{l} \in \mathrm{GL}_{p}(k)$ and commuting $B_{1}, \ldots, B_{l} \in \mathrm{GL}_{q}(k)(p, q \geq 1)$. This addition rule is not expressed by a continuous map $\operatorname{Comm}_{l}(k) \times$ $\mathrm{Comm}_{l}(k) \rightarrow \operatorname{Comm}_{l}(k)$, but the following continuous map $\mathrm{Comm}_{l}(k) \times \operatorname{Comm}_{l}(k) \rightarrow \operatorname{Comm}_{l}(k)$ actually induces the group operation on $K_{l}^{M}(k)$ :

$$
\begin{aligned}
& \left(\left(\left(\begin{array}{cccc}
a_{11} & a_{12} & a_{13} & \cdots \\
a_{21} & a_{22} & a_{23} & \cdots \\
a_{31} & a_{32} & a_{33} & \cdots \\
\vdots & \vdots & \vdots & \ddots
\end{array}\right), \ldots\right)\right. \\
& \left(\left(\begin{array}{cccc}
b_{11} & b_{12} & b_{13} & \cdots \\
b_{21} & b_{22} & b_{23} & \cdots \\
b_{31} & b_{32} & b_{33} & \cdots \\
\vdots & \vdots & \vdots & \ddots
\end{array}\right), \ldots\right)
\end{aligned}
$$



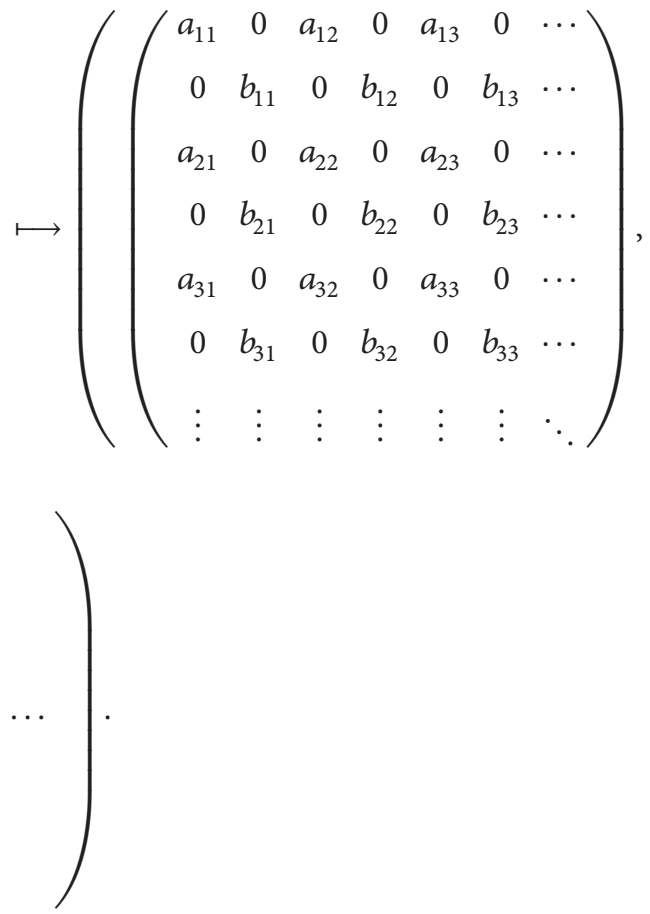

To prove that the two elements

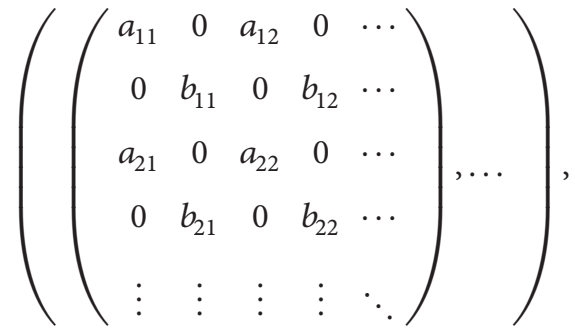

$$
\left(\left(\begin{array}{cccccc}
a_{11} & a_{12} & \cdots & 0 & 0 & \cdots \\
a_{21} & a_{22} & \cdots & 0 & 0 & \cdots \\
\vdots & \vdots & \ddots & \vdots & \vdots & \vdots \\
0 & 0 & \cdots & b_{11} & b_{12} & \cdots \\
0 & 0 & \cdots & b_{21} & b_{22} & \cdots \\
\vdots & \vdots & \cdots & \vdots & \vdots & \ddots
\end{array}\right), \cdots\right.
$$

of $\operatorname{Comm}_{l}(k)$ map to the same element under $\Phi_{l}$ : $\mathrm{Comm}_{l}(k) \rightarrow K_{l}^{M}(k)$, it is enough to verify that an $l$-tuple $\left(A_{1}, \ldots, A_{l}\right)$ of commuting matrices in $\mathrm{GL}_{n}(k)^{l}$ represents the same element in $\mathrm{GW}_{l}(k)$ which is represented by the $l$-tuple of matrices which is obtained by simultaneously changing $i$ th and $j$ th rows and also $i$ th and $j$ th columns of all $l n \times$ $n$ matrices $A_{1}, \ldots, A_{l}$. For notational convenience, we will prove this for 1 st and 2 nd rows and columns of $2 \times 2$ matrices and the proof is easily generalized to $n \times n$ matrices. Let us write the $(i, j)$ th entry of the matrix $A_{k}$ as $a_{i j}^{k}(k=1,2, \ldots, l)$. In $\mathrm{GW}_{l}(k)$, we have

$$
\begin{aligned}
& \left(A_{1}, \ldots, A_{l}\right)=\left(\left(\begin{array}{ll}
a_{11}^{1} & a_{12}^{1} \\
a_{21}^{1} & a_{22}^{1}
\end{array}\right), \ldots,\left(\begin{array}{ll}
a_{11}^{l} & a_{12}^{l} \\
a_{21}^{l} & a_{22}^{l}
\end{array}\right)\right) \\
& \quad=\left(\left(\begin{array}{cccc}
a_{11}^{1} & a_{12}^{1} & 0 & 0 \\
a_{21}^{1} & a_{22}^{1} & 0 & 0 \\
0 & 0 & 1 & 0 \\
0 & 0 & 0 & 1
\end{array}\right), \ldots,\right. \\
& \left.\left(\begin{array}{cccc}
a_{11}^{l} & a_{12}^{l} & 0 & 0 \\
a_{21}^{l} & a_{22}^{l} & 0 & 0 \\
0 & 0 & 1 & 0 \\
0 & 0 & 0 & 1
\end{array}\right)\right)
\end{aligned}
$$

Using the polynomial homotopy

$$
\begin{aligned}
& \left(\begin{array}{cccc}
1-t^{2} & 0 & 0 & t \\
0 & 1 & 0 & 0 \\
0 & 0 & 1 & 0 \\
t^{3}-2 t & 0 & 0 & 1-t^{2}
\end{array}\right)\left(\begin{array}{cccc}
a_{11}^{1} & a_{12}^{1} & 0 & 0 \\
a_{21}^{1} & a_{22}^{1} & 0 & 0 \\
0 & 0 & 1 & 0 \\
0 & 0 & 0 & 1
\end{array}\right)\left(\begin{array}{cccc}
1-t^{2} & 0 & 0 & -t \\
0 & 1 & 0 & 0 \\
0 & 0 & 1 & 0 \\
2 t-t^{3} & 0 & 0 & 1-t^{2}
\end{array}\right), \ldots \\
& \left(\begin{array}{cccc}
1-t^{2} & 0 & 0 & t \\
0 & 1 & 0 & 0 \\
0 & 0 & 1 & 0 \\
t^{3}-2 t & 0 & 0 & 1-t^{2}
\end{array}\right)\left(\begin{array}{cccc}
a_{11}^{l} & a_{12}^{l} & 0 & 0 \\
a_{21}^{l} & a_{22}^{l} & 0 & 0 \\
0 & 0 & 1 & 0 \\
0 & 0 & 0 & 1
\end{array}\right)\left(\begin{array}{cccc}
1-t^{2} & 0 & 0 & -t \\
0 & 1 & 0 & 0 \\
0 & 0 & 1 & 0 \\
2 t-t^{3} & 0 & 0 & 1-t^{2}
\end{array}\right),
\end{aligned}
$$


which results in interchanging the 1st and 4th rows with negative sign to the new 4 th row and then interchanging 1st and 4 th columns with negative sign to the new 4 th column, we see that, in $\mathrm{GW}_{l}(k)$,

$$
\left(\left(\begin{array}{cccc}
a_{11}^{1} & a_{12}^{1} & 0 & 0 \\
a_{21}^{1} & a_{22}^{1} & 0 & 0 \\
0 & 0 & 1 & 0 \\
0 & 0 & 0 & 1
\end{array}\right), \ldots,\left(\begin{array}{cccc}
a_{11}^{l} & a_{12}^{l} & 0 & 0 \\
a_{21}^{l} & a_{22}^{l} & 0 & 0 \\
0 & 0 & 1 & 0 \\
0 & 0 & 0 & 1
\end{array}\right)\right)
$$

$$
\begin{aligned}
& =\left(\left(\begin{array}{cccc}
1 & 0 & 0 & 0 \\
0 & a_{22}^{1} & 0 & -a_{21}^{1} \\
0 & 0 & 1 & 0 \\
0 & -a_{12}^{1} & 0 & a_{11}^{1}
\end{array}\right), \ldots\right. \\
& \left.\left(\begin{array}{cccc}
1 & 0 & 0 & 0 \\
0 & a_{22}^{l} & 0 & -a_{21}^{l} \\
0 & 0 & 1 & 0 \\
0 & -a_{12}^{l} & 0 & a_{11}^{l}
\end{array}\right)\right)
\end{aligned}
$$

Again, by applying the polynomial homotopy

$$
\begin{aligned}
& \left(\begin{array}{cccc}
1 & 0 & 0 & 0 \\
0 & 1-t^{2} & t & 0 \\
0 & t^{3}-2 t & 1-t^{2} & 0 \\
0 & 0 & 0 & 1
\end{array}\right)\left(\begin{array}{cccc}
1 & 0 & 0 & 0 \\
0 & a_{22}^{1} & 0 & -a_{21}^{1} \\
0 & 0 & 1 & 0 \\
0 & -a_{12}^{1} & 0 & a_{11}^{1}
\end{array}\right)\left(\begin{array}{cccc}
1 & 0 & 0 & 0 \\
0 & 1-t^{2} & -t & 0 \\
0 & 2 t-t^{3} & 1-t^{2} & 0 \\
0 & 0 & 0 & 1
\end{array}\right), \ldots \\
& \left.\left(\begin{array}{cccc}
1 & 0 & 0 & 0 \\
0 & 1-t^{2} & t & 0 \\
0 & t^{3}-2 t & 1-t^{2} & 0 \\
0 & 0 & 0 & 1
\end{array}\right)\left(\begin{array}{cccc}
1 & 0 & 0 & 0 \\
0 & a_{22}^{l} & 0 & -a_{21}^{l} \\
0 & 0 & 1 & 0 \\
0 & -a_{12}^{l} & 0 & a_{11}^{l}
\end{array}\right)\left(\begin{array}{cccc}
1 & 0 & 0 & 0 \\
0 & 1-t^{2} & -t & 0 \\
0 & 2 t-t^{3} & 1-t^{2} & 0 \\
0 & 0 & 0 & 1
\end{array}\right)\right)
\end{aligned}
$$

which results in interchanging the 2 nd and 3 rd rows with negative sign to the new 3rd row and then interchanging 2 nd and 3rd columns with negative sign to the new 3rd column, we have, in $\mathrm{GW}_{l}(k)$,

$$
\begin{aligned}
& \left(\left(\begin{array}{cccc}
1 & 0 & 0 & 0 \\
0 & a_{22}^{1} & 0 & -a_{21}^{1} \\
0 & 0 & 1 & 0 \\
0 & a_{12}^{1} & 0 & a_{11}^{1}
\end{array}\right), \ldots,\left(\begin{array}{cccc}
1 & 0 & 0 & 0 \\
0 & a_{22}^{l} & 0 & -a_{21}^{l} \\
0 & 0 & 1 & 0 \\
0 & a_{12}^{l} & 0 & a_{11}^{l}
\end{array}\right)\right) \\
& =\left(\left(\begin{array}{cccc}
1 & 0 & 0 & 0 \\
0 & 1 & 0 & 0 \\
0 & 0 & a_{22}^{1} & a_{21}^{1} \\
0 & 0 & a_{12}^{1} & a_{11}^{1}
\end{array}\right), \ldots,\right. \\
& \left.\left(\begin{array}{llll}
1 & 0 & 0 & 0 \\
0 & 1 & 0 & 0 \\
0 & 0 & a_{22}^{l} & a_{21}^{l} \\
0 & 0 & a_{12}^{l} & a_{11}^{l}
\end{array}\right)\right),
\end{aligned}
$$

which is equal to $\left(\left(\begin{array}{cc}a_{22}^{1} & a_{21}^{1} \\ a_{12}^{1} & a_{11}^{1}\end{array}\right), \ldots,\left(\begin{array}{cc}a_{22}^{l} & a_{21}^{l} \\ a_{12}^{l} & a_{11}^{l}\end{array}\right)\right)$ in $\mathrm{GW}_{l}(k)$.

\section{The Topological Structures of $K_{l}^{M}(\mathbb{R})$ and $K_{l}^{M}(\mathbb{C})$}

Theorem 3. For $l \geq 2$, the topological space $K_{l}^{M}(\mathbb{R})$ is a disjoint union of two indiscrete open sets.

Proof. Note that we have $K_{l}^{M}(\mathbb{R}) \simeq(\mathbb{Z} / 2) \oplus H$, where the first direct factor $\mathbb{Z} / 2$ is generated by $\{-1, \ldots,-1\}$ and $H$ is a uniquely divisible group [2]. For $\left\{a_{1}, \ldots, a_{l}\right\}$ where $a_{i}$ are negative for all $i=1, \ldots, l$, we have $\left\{a_{1}, \ldots, a_{l}\right\}=$ $\left\{-1, a_{2}, \ldots, a_{l}\right\}+\left\{-a_{1}, a_{2}, \ldots, a_{l}\right\}=\left\{-1,-1, a_{3}, \ldots, a_{l}\right\}+$ $\left\{-1,-a_{2}, \ldots, a_{l}\right\}+\left\{-a_{1}, a_{2}, \ldots, a_{l}\right\}=\cdots$ which is equal to the sum of $\{-1,-1,-1, \ldots,-1\}$ and various symbols of the form $\left\{b_{1}, \ldots, b_{l}\right\}$ where at least one of $b_{i}$ is positive.

Every element of $H$ can be written as a sum of symbols of the form $b_{1}, \ldots, b_{l}$, where at least one of $b_{i}$ is positive. By writing a positive real number as a square of its square root, we may assume that $b_{i}$ is positive for every $i=1, \ldots, l$ (e.g., $\left\{b_{1}, b_{2}\right\}=\left\{b_{1}^{2}, \sqrt{b_{2}}\right\}$ in case $b_{2}>0$ ).

Let $U$ be any open set of $K_{l}^{M}(\mathbb{R})$ containing the identity element and consider its inverse image $V=$ $\Phi_{l}^{-1}(U)$ in $\operatorname{Comm}_{l}(\mathbb{R})$. Let $h \in H$ be any element. Then 
$\Phi_{l}^{-1}(\{h\})$ contains an $l$-tuple of diagonal matrices of the form

$$
\left(\left(\begin{array}{cccc}
a_{1}^{1} & & & \\
& a_{2}^{1} & & \\
& & \ddots & \\
& & & a_{n}^{1}
\end{array}\right), \ldots,\left(\begin{array}{cccc}
a_{1}^{l} & & & \\
& a_{2}^{l} & & \\
& & \ddots & \\
& & & a_{n}^{l}
\end{array}\right)\right),
$$

where $a_{k}^{i}$ is positive for every $k=1, \ldots, n$ and $i=1, \ldots, l$. By taking $m$ th root of $a_{k}^{1}$ for sufficiently large $m$, we may assume that $a_{k}^{1}$ is arbitrarily close to 1 . Then $\Phi_{l}^{-1}(\{h\})$ contains an element which is arbitrarily close to $\left(A_{1}, \ldots, A_{l}\right)$ with $A_{1}$ equal to the identity matrix. So, $\Phi_{l}^{-1}(\{h\})$ contains an element which is contained in the open set $V=\Phi_{l}^{-1}(U)$. Hence $U$ must contain $H$.

Similarly, the coset $H+\{-1,-1, \ldots,-1\}$ is also an indiscrete subspace. In fact, $H$ is the image under $\Phi_{l}$ of the set of l-tuples $\left(A_{1}, \ldots, A_{l}\right)$ of commuting matrices in $\mathrm{GL}_{n}(\mathbb{R})^{l}(n \geq$ 1) such that the determinants of $A_{i}$ are positive for some $i \in$ $\{1, \ldots, l\}$. On the other hand, $H+\{-1,-1, \ldots,-1\}$ is the image under $\Phi_{l}$ of the set of $l$-tuples $\left(A_{1}, \ldots, A_{l}\right)$ of commuting matrices where the determinants of $A_{i}$ are negative for all $i=1, \ldots, l$. Therefore, the proper open sets of $K_{l}^{M}(\mathbb{R})$ are $H$ and $H+\{-1,-1, \ldots,-1\}$.

Corollary 4. For $l \geq 2$, the topological space $K_{l}^{M}(\mathbb{C})$ is indiscrete (trivial).

Proof. Let $U$ be an open set of $K_{l}^{M}(\mathbb{C})$ containing the identity element and let $V=\Phi^{-1}(U) \subset \operatorname{Comm}_{l}(\mathbb{C})$. For any element $h \in K_{l}^{M}(\mathbb{C}), \Phi^{-1}(\{h\})$ contains an $l$-tuple $\left(A_{1}, \ldots, A_{l}\right)$ of diagonal matrices. Write each diagonal element of $A_{i}$ as a product of a positive real number and a complex number with absolute value 1 . Any complex number with absolute value 1 is arbitrarily close to a root of unity and any symbol containing a root of unity is trivial since, for example, $\{\zeta, b\}=$ $(1 / m)\left\{\zeta^{m}, b\right\}=0$ if $\zeta$ is an $m$ th root of unity. Combining this fact with the arguments given in the proof of Theorem 3, we see that $\Phi^{-1}(\{h\})$ contains an element which is contained in $V=\Phi^{-1}(U)$. This shows that the natural topology on $K_{l}^{M}(\mathbb{C})$ is indiscrete.

\section{Applications to Joint Determinants}

When $k$ is a topological field, a joint determinant from $\mathrm{Comm}_{l}(k)$ into a topological abelian group $G$ is called continuous if $\operatorname{Comm}_{l}(k)$ is given the subspace topology of $\mathrm{GL}(k)=\cup_{n \rightarrow \infty} \mathrm{GL}_{n}(k)$ with the direct limit topology as described in Section 2. Since the natural topology on $K_{l}^{M}(k)$ in Definition 1 is the quotient topology, any continuous joint determinant induces a continuous map from $K_{l}^{M}(k)$ into $G$ and vice versa.

Corollary 5. For $l \geq 2$ and any topological abelian group $G$ which is $T_{0}$, any continuous joint determinant from $\operatorname{Comm}_{l}(\mathbb{R})$ into $G$ factors through the discrete group $\mathbb{Z}_{2}$.
Proof. This follows directly from Theorem 3 . Note that a topological group is Hausdorff if it is $T_{0}$ (cf. Lemma 10.1 of [5]).

The following is a direct consequence of Corollary 4.

Corollary 6. For $l \geq 2$ and any topological abelian group $G$ which is $T_{0}$, any continuous joint determinant from $\operatorname{Comm}_{l}(\mathbb{C})$ into $G$ is trivial.

We summarize our results on the continuous joint determinants for $k=\mathbb{R}$ and $k=\mathbb{C}$ in the following theorem, which is virtually equivalent to Theorem 3 and Corollary 4 .

Theorem 7. For $k=\mathbb{R}$ or $k=\mathbb{C}$ and a topological abelian group $G$, let $D: \operatorname{Comm}_{l}(k) \rightarrow G$ be a continuous surjective joint determinant. When $l=1, D$ is a composite of the usual determinant map followed by a canonical epimorphism $k^{*} \rightarrow$ $G$ with $G$ equipped with a coarser topology than the quotient topology induced by the epimorphism. When $k=\mathbb{R}$ and $l \geq 2$, $G$ either is an indiscrete space or has an indiscrete subgroup of index 2 . If $k=\mathbb{C}$, then $G$ has the indiscrete topology.

\section{Conflicts of Interest}

The author declares that they have no conflicts of interest.

\section{Acknowledgments}

This work was supported by the National Research Foundation of Korea (NRF) grant funded by the Korean government (MEST) (no. NRF-2010-0006083).

\section{References}

[1] S. Myung, "Transfer maps and nonexistence of joint determinant," Linear Algebra and its Applications, vol. 431, no. 9, pp. 1633-1651, 2009.

[2] J. Milnor, "Algebraic $K$-theory and quadratic forms," Inventiones Mathematicae, vol. 9, pp. 318-344, 1969/1970.

[3] V. Voevodsky, "On motivic cohomology with Z/l-coefficients," Annals of Mathematics: Second Series, vol. 174, no. 1, pp. 401438, 2011.

[4] F. Paul, J. BaumRubén, and a. Sánchez-Garc, "K-theory for group $\mathrm{C}^{*}$-algebras," in Topics in Algebraic and Topological KTheory, pp. 1-43, Springer, Berlin, Germany, 2008.

[5] M. F. Atiyah and I. G. Macdonald, Introduction to Commutative Algebra, Addison-Wesley Publishing Co., London, UK, 1969. 


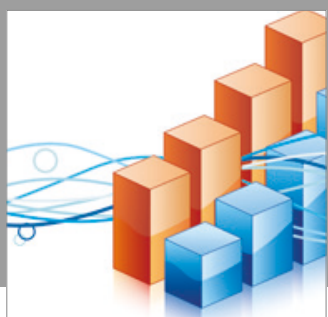

Advances in

Operations Research

vatersals

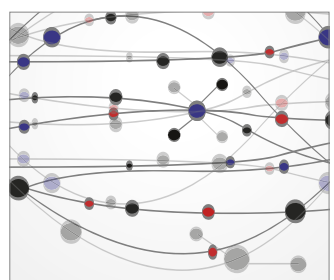

\section{The Scientific} World Journal
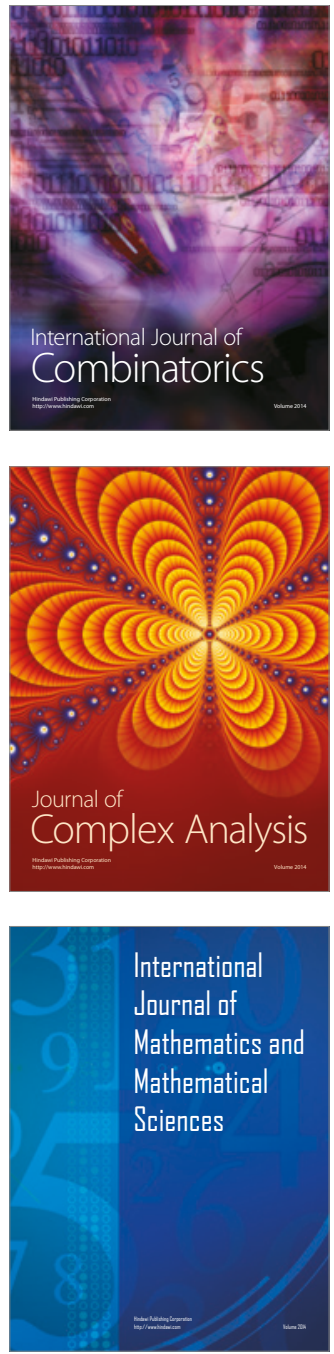
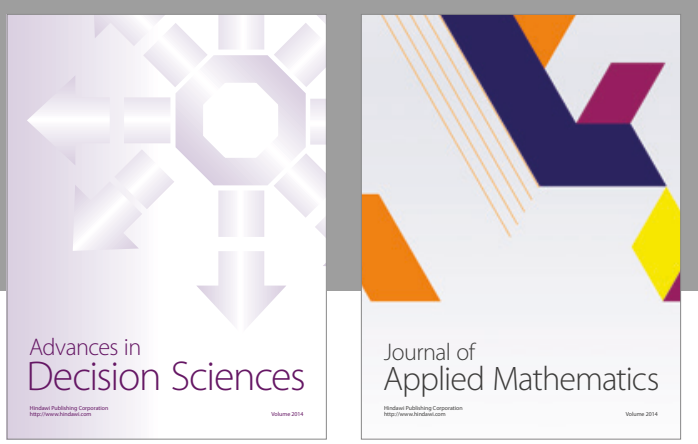

Algebra

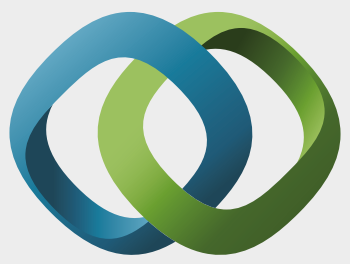

\section{Hindawi}

Submit your manuscripts at

https://www.hindawi.com
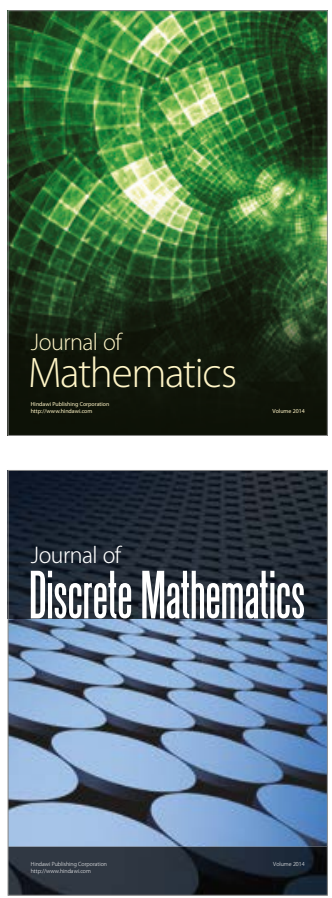

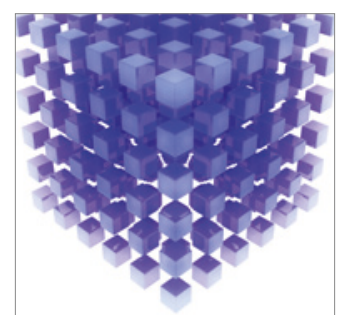

Mathematical Problems in Engineering
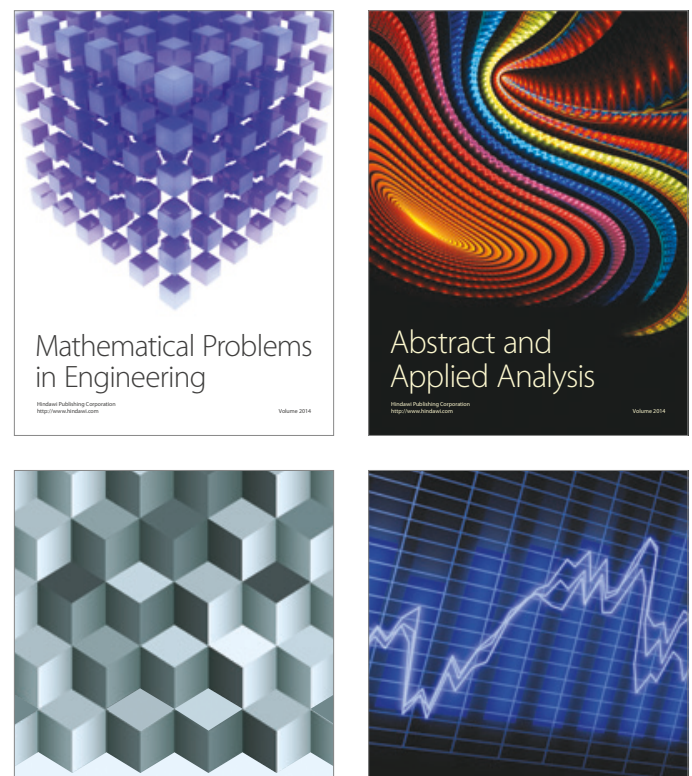

Journal of

Function Spaces

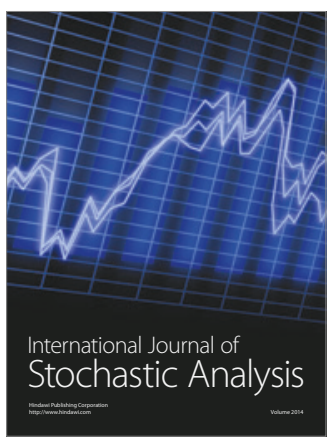

Probability and Statistics
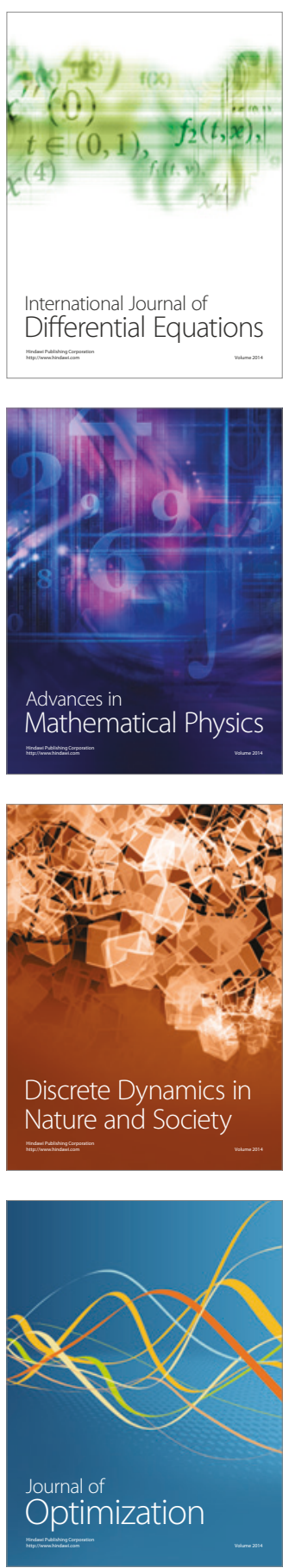